\title{
COMBINATORIAL STRUCTURES IN SPIN MODELS: ALGORITHMS FOR GENERATION OF OPERATOR MATRICES
}

\author{
WOJCIECH FLOREK \\ Adam Mickiewicz University, Institute of Physics \\ Umultowska 85, 61-614 Poznań, Poland \\ e-mail: $\quad$ florek@spin.amu.edu.pl
}

\begin{abstract}
Two combinatorial algorithms, generation of ordered partitions of $N$ with no more than $m$ parts and decompositions of $N$-element set into subsets with cardinalities given by a partition $[k]=\left[k_{0} k_{1} \ldots k_{\mathrm{m}-1}\right]$ are presented and their possible applications to finite (mesoscopic) spin systems are indicated. The flow charts, listings, and results of test runs are provided.
\end{abstract}

\section{INTRODUCTION}

In the previous paper [1] a method of solving an eigen problem of a Hamiltonian acting in a finite space of states were presented. This method is based on the earlier article [2], where an expression for operator matrix elements was formulated. Application of this formula needs some group-theoretical and combinatorial structures to be introduced and then generated. Some steps can be carried out with use of standard procedures, however two of them (the generation of partitions of $N$ with no more than $m$ parts and the decomposition of $N$-element set into $l p \leq m$ nonempty subsets with cardinalities $k_{0} \geq k_{1} \geq \ldots \geq k_{l p-1}>0$ ) require new solutions - at least modifications of the standard procedures. These tasks are related, since ordered partitions $[\mathrm{k}]=$ $\left[k_{0}, k_{1}, \ldots, k_{m-1}\right]$ of $N$ into $m$ non-negative parts generated in the first step are input data in the second step. The main ideas of solutions proposed here were presented in the previous work [1], whereas this paper is devoted to more detailed discussion.

Though algorithms have been implemented in FORTRAN (F77 and F90) and C, they are presented below in the Pascal-like convention (without semi-colons), what is more readable. Some bunches of standard instructions are replaced by one command (especially the "print" command), since they are not important to the presentation of algorithms. The flow charts are also included. The last implementation and compilation were done under Linux (Red Hat 6.2) with egcs-2.91.66 on the Pentium 300 MHZ PC and the provided timings have been obtained on this machine.

\section{ORDERED PARTITIONS OF $N$}

\section{WITH NO MORE THAN $m$ NONZERO PARTS}

One of algorithms for the generation of partitions produces them in the antilexicographic order, i.e. a partition $[k]$ is generated before a partition $\left[k^{\prime}\right]$ if a word $k_{0}^{\prime} k_{1}^{\prime} \ldots k_{m}^{\prime}$, precedes lexicographically a word $k_{0} k_{1} \ldots k_{m^{\prime}}$. It means that there exists $\operatorname{such} 0 \leq l \leq \min \left(m, m^{\prime}\right)$ that $k_{i}=$ $k_{I}^{\prime}$ for $I<l$ and $k_{l}^{\prime}<k_{l}$. This algorithm makes a use of two arrays: $S[m]$ containing pairwise different parts and $R[m]$ with multiplicities (repetitions) of these parts $[3,4]$, so it is easy to print 
out partitions in the notion $\left[s_{0}^{r_{0}}, s_{1}^{r_{1}}, \ldots, s_{m-1}^{r_{m-1}}\right]$. The algorithm presented below exploits the notion of associated partitions [5], Formally, the partition [ $k$ '] associated with $[k]$ is determined by the following formula:

$$
k_{l}^{\prime}:=\sum_{i \mid k_{i}>l} 1, \quad 0 \leq l<N .
$$

Introducing the multiplicities $R[j]$ this definition can be rewritten as [5]:

$$
k_{l}^{\prime}:=\sum_{i=l+l}^{N} R[i], \quad 0 \leq l<N .
$$

For example, partitions $[k]=\left[6,4^{2}, 2\right]$ and $\left[k^{\prime}\right]=\left[4^{2}, 3^{2}, 1^{2}\right]$ are associated, since we have:

$$
\begin{array}{lll}
k_{0}^{\prime}=\sum_{i=0}^{3} 1=4, & k_{1}^{\prime}=\sum_{i=0}^{3} 1=4, & k_{2}^{\prime}=\sum_{i=0}^{2} 1=3, \\
k_{3}^{\prime}=\sum_{i=0}^{2} 1=3, & k_{4}^{\prime}=\sum_{i=0}^{0} 1=1, & k_{5}^{\prime}=\sum_{i=0}^{0} 1=1,
\end{array}
$$

on the one hand, and

$$
\begin{array}{ll}
k_{0}=\sum_{i=1}^{16} R^{\prime}[i]=6, & k_{1}=\sum_{i=2}^{16} R^{\prime}[i]=4, \\
k_{2}=\sum_{i=3}^{16} R^{\prime}[i]=4, & k_{3}=\sum_{i=4}^{16} R^{\prime}[i]=2,
\end{array}
$$

on the other. In order to generate partitions with no more than $m$ parts the standard algorithm is used but for each generated partitions its associated counter-part is printed out. Hence, the array $R$ is preserved in the algorithm proposed (but now is called $m u l$ ). However, for the further purposes it is more convenient to have partitions written as vectors $\left[\mathrm{k}_{0}, \mathrm{k}_{1}, \ldots, \mathrm{k}_{\mathrm{m}-1}\right]$, so the array $S$ is replaced by an array $\operatorname{par}[m]$ and, therefore, some new modification rules are necessary. It needs introductions of two parameters, $f p$ and $l p$, related to the first and the last part of the original (not associated) partition, respectively. During the generation these indices can reach any value from the interval $[0, \boldsymbol{m})$, but for control purposes they can be also negative $(-1$, in fact). A flow chart of the proposed algorithm is presented in Fig. 1. The block "err" controls read parameters and stops the program if imposed limits are broken (too large or too small $\boldsymbol{N}$ and $\boldsymbol{m}$ ). The other parts are briefly described below and their listings are presented.

In the first block all arrays and variables are initialized. It is easy to notice that the first printed partition consists of at most two different numbers: $[\mathrm{N} / \mathrm{m}]=j d+1$ and $[\mathrm{N} / \mathrm{m}]=j d$. If $j m=N \bmod$ $m$ than the multiplicities of these parts are $j m$ and $m-j m$, respectively. Hence the associated (and generated, in fact) partition has $j d$ parts equal $m$ and one part equal jm. Therefore, in line 06 we have $m u l[0]:=j d$ and the array par is initialized in the line 08 . Since at this moment a remainder is unknown, then the first and last parts are the same (see line 07). Next the first jm parts have to be increased and, simultaneously, $m u l[l p]$ has to be set to 1 . (Note that $m u l[0]$ need not to be modified). The index $l p$ is determined relatively with respect to $m$ : since 0 corresponds to $m$ 
Fig. 1. Partition generation

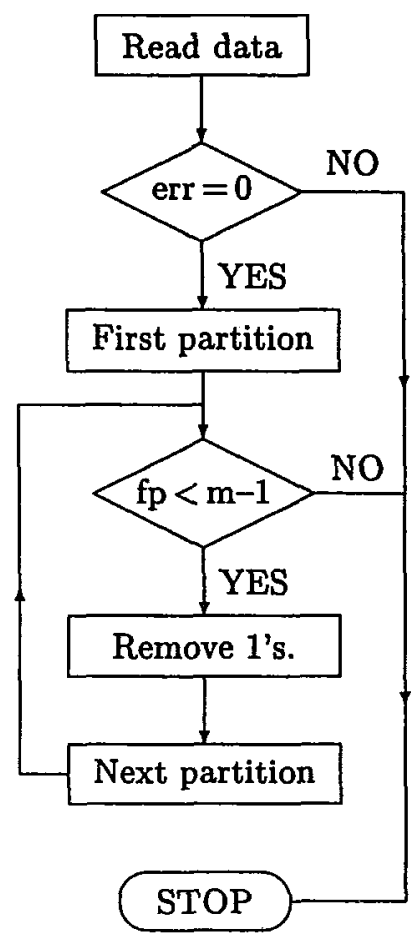

01 First partition\}

$02 f p:=-1$

03 lp $:=-1 \quad\{$ It may be ommited. $\}$

$04 j d:=N \operatorname{div} m$

05 if $(j d>0)$ begin

$06 m u l[0]:=j d$

$07 \quad l p:=f p:=0$

08 for $I:=0$ to $m-1$ do $\operatorname{par}[i]:=j d$

09 end

$10 \mathrm{jm}:=N \bmod m$

11 if $(j m>0)$ begin

$12 \quad l p:=m-j m$

13 if $(f p<0) f p:=l p$

$14 m u l[l p]:=1$

$15 j d:=j d+1$

16 for $I:=0$ to $j m-1$ do $\operatorname{par}[i]:=j d$

17 end

18 print (par) 
19 sum $:=0$

20 \{Remove l's.\}

21 if $(l p=m-1)$ begin

22 sum: $:=\operatorname{mul}[m-1]$

$23 \operatorname{par}[0]:=\operatorname{par}[0]-$ sum

$24 m u l[m-1]:=0$

25 repeat $l p:=l p-1$ until $(m u l[l p] \diamond 0)$

26 end

27 NNext partition\}

$28 j d:=m-l p$

29 sum $:=$ sum $+j d$

$30 j d:=j d-1$

$31 m u l[l p]:=m u l[l p]-1$

32 if $((l p=f p)$ and $(m u l[l p]=0)) f p:=-1$

33 for $I:=0$ to $j d$ do $\operatorname{par}[i]:=\operatorname{par}[i]-1$

$34 l p:=l p+1$

35 if $(f p<0) f p:=l p$

$36 \mathrm{jm}:=$ sum div $j d$

$37 \mathrm{mul}[\mathrm{lp}]:=\mathrm{jm}$

38 for $I:=0$ to $j d-1$ do $\operatorname{par}[i]:=\operatorname{par}[i]+j m$

$39 \mathrm{jm}:=\operatorname{sum} \bmod j d$

40 if $(j m>0)$ begin

$41 \quad l p:=m-j m$

$42 \operatorname{mul}[l p]:=1$

43 for $l:=0$ to $j m-1$ do $\operatorname{par}[i]=\operatorname{par}[i]+1$

44 end

45 print (par)

Fig. 3. Generation of the consecutive partitions

(the first part of generated partition) then $\boldsymbol{j} \boldsymbol{m}$ (the last part, respectively) corresponds to $\boldsymbol{m} \boldsymbol{-} \boldsymbol{j} \boldsymbol{m}$. For example, when $\boldsymbol{N}=\mathbf{7}$ and $\boldsymbol{m}=\mathbf{3}$, then $\boldsymbol{j} \boldsymbol{d}=2$ and $\boldsymbol{j} \boldsymbol{m}=1$, so after the first step one obtains:

$$
[\operatorname{mul}[0]=2, \quad \text { par }=[2,2,2], \mathrm{fp}=\mathrm{lp}=0]
$$

what is modified in the second step to

$$
[\operatorname{mul}[2]=1, \quad \text { par }=[3,2,2], \quad f p=0, \quad \text { lp }=2]
$$

and the associated partition equals $[3,3,1]$, i.e. the first (antilexicographically) partition of 7 with the first part equal to 3 .

The generation of partitions ends when all parts equal 1 , i.e. $[k]=\left[1^{\mathrm{N}}\right]$ and $\left[\boldsymbol{k}^{\prime}\right]=[N, 0, \ldots, 0]$, So, in the present modification it corresponds to the condition $f \boldsymbol{p}=\boldsymbol{m}-1$. In the other case the 
next partition is generated. At first all parts equal 1 are removed. Such parts appear in $[\mathrm{k}]$ if $\boldsymbol{l} \boldsymbol{p}=\boldsymbol{m} \boldsymbol{-} 1$ or, in the other words, $\boldsymbol{k}_{\boldsymbol{0}} \boldsymbol{>}_{\boldsymbol{k}_{\boldsymbol{l}}}$. Of course $\boldsymbol{m u l}[\boldsymbol{m}-1]$ has to be set to zero and the new last part has to be determined. It can be done by a repeat... until loop, as in the presented listing, or by calculating a number of parts equal to par[0] (it is at least two, since par[0] =par[1] now). Removed 1 's and the last part $\boldsymbol{j} \boldsymbol{d}=\boldsymbol{m}$ - $\boldsymbol{p} \boldsymbol{p}$ are summed up (see the line 29) and decomposed into $\boldsymbol{j} \boldsymbol{d}$ - 1 parts in a similar way as $\boldsymbol{N}$ was decomposed into $\boldsymbol{m}$ parts in the first partition. For $\boldsymbol{N}=7$ and $m=3$ this algorithm generates the following eight partitions (below they are presented with their associated counter-parts):
$[3,2,2] \quad[3,3,1]$
$[3,3,1] \quad[3,2,2]$
$[4,2,1] \quad[3,2,1,1]$
$[5,1,1] \quad[3,1,1,1,1]$
$[4,3,0] \quad[2,2,2,1]$
$[5,2,0] \quad[2,2,1,1,1]$
$[6,1,0] \quad[2,1,1,1,1,1]$
$[7,0,0] \quad[1,1,1,1,1,1,1]$

The results obtained on the Pentium 300 MHZ machine are presented in Table I. The timings are calculated as mean values of ten through million (for small values of $\boldsymbol{N}$ and $\boldsymbol{m}$ ) runs of a test program without control and printing blocks. The cases when the number of partitions is greater than $2^{32}$ have not been tested. A number of steps necessary to generate a new partition is independent of $\boldsymbol{N}$ and $\mathrm{m}$, as in the standard procedure [3,4], The visible tendency of a bit faster generation for large $\boldsymbol{N}$ and small $\boldsymbol{m}$ is caused by shorter test loops for large $\boldsymbol{N}$ and shorter substitution loops for smaller $\mathrm{m}$ (lines $33,38,43$ ).

Table I. Number of partitions NP and generation speed (partitions per second) for some values of $N$ and $m$

\begin{tabular}{rcrrrrr}
\hline$N$ & $m$ & \multicolumn{1}{c}{3} & \multicolumn{1}{c}{4} & \multicolumn{1}{c}{5} & \multicolumn{1}{c}{6} & \multicolumn{1}{c}{7} \\
\hline 100 & $N P$ & 884 & 8,037 & 46,262 & 189,509 & 596,763 \\
& speed & $2,448,753$ & $2,417,916$ & $2,384,640$ & $2,368,862$ & $2,313,034$ \\
200 & $N P$ & 3,434 & 59,823 & 643,287 & $4,775,383$ & $26,366,879$ \\
& speed & $2,464,456$ & $2,456,156$ & $2,450,617$ & $2,430,671$ & $2,413,949$ \\
400 & $N P$ & 13,534 & 461,312 & $9,572,962$ & $134,851,969$ & $1,386,102,973$ \\
& speed & $2,506,296$ & $2,509,872$ & $2,497,294$ & $2,487,613$ & $2,483,237$ \\
700 & $N P$ & 41,184 & $2,433,337$ & $86,994,037$ & $2,095,277,554$ & $>2^{32}$ \\
& speed & $2,511,219$ & $2,509,872$ & $2,505,207$ & $2,485,834$ & n.a. \\
1000 & $N P$ & 83,834 & $7,049,112$ & $357,746,987$ & $>2^{32}$ & $>2^{32}$ \\
& speed & $2,521,323$ & $2,517,540$ & $2,515,349$ & n.a. & n.a. \\
\hline
\end{tabular}




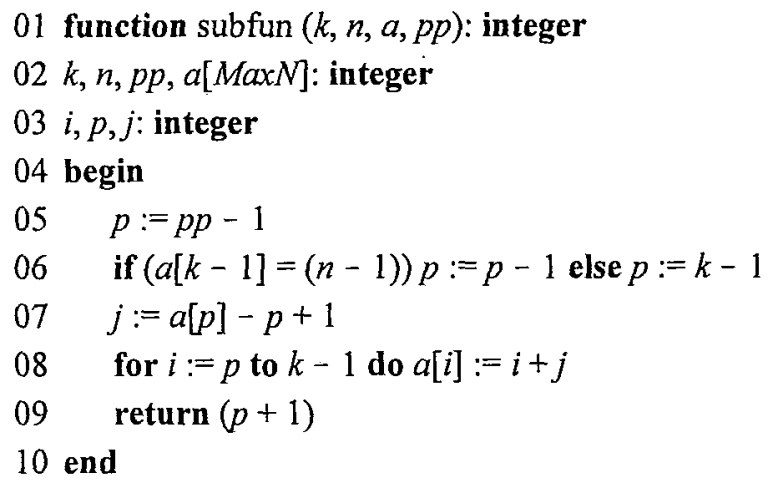

Fig. 4. Modified procedure of subsets generation

\section{DECOMPOSITION OF $N$-ELEMENT SET INTO $m$ SUBSETS}

There are some algorithms to generate $k$-element subsets of $N$-element set [3,4], however it seems they can not be modified to generate $m$ subsets of cardinalities $k_{0}, k_{1}, \ldots, k_{m-1}$, where $[k]$ is a partition of $N$. The proposed solution is based on an algorithm generating $k$-element subsets in the lexicographic order, i.e. it starts from $\{0,1, \ldots, k-1\}$ and goes through $\{0,1, \ldots, k-2, k\}$, $\ldots,\{0,1, \ldots, k-2, n\}$ to $\{n-k, \ldots, n-1\}$. This algorithm is applied at different levels to many A'element sets and their $i$-element subsets, so it has been included as a function. Two parameters, the cardinalities of a set and a subset, are transferred to the function "by value", whereas the other two - "by address" (the subset $a[k]$ and the so-called moving point $p p$ ). In the actual implementation $p$ is returned as the function value. Moreover, some solutions in the main program imply that the moving point in the function has to be decreased (line 05 and return $(p+1)$ in line 09).

Since each partition $[k]$ of $N$ can be represented by an ordered one, then the algorithm can be limited to this case. In the previous section ordered partitions into $m$ non-negative parts (trailing zeroes are not truncated) has been considered, so they will be treated as the input data. Therefore, in the first step a number of non-zero parts $(l p)$ has to be determined. The main idea of the proposed algorithm consists in decomposition of the generation process in such steps that at each moment one subset is generated with use of the standard algorithm. Let $[k]=\left[k_{0}, k_{1}, \ldots, k_{m-1}\right]$ be an ordered partition of $N$ into $m$ non-zero parts. Then to generate $m$ subsets of cardinalities $k_{0}$, $k_{1}, \ldots, k_{m-1}$ one starts with $k_{\mathrm{m}-1}$-element subsets of $N=N_{m-1}$, where. $N_{l}=\Sigma_{l=0}^{l} k_{i}$ and $l=0,1, \ldots$, $m$-1. In general, in the $l^{\text {th }}$ step, one generates $k_{l}$-element subsets of $N_{l}$-element set, where $l=1$, $2, \ldots, m-1$. Let us consider a partition $[k]=[3,2,2]$ generated by the previous algorithm. So one starts with two-element subsets of a set with seven elements $0,1, \ldots, 6$. Of course, there are 21 subsets, $\{0,1\},\{0,2\}, \ldots,\{0,6\},\{1,2\}, \ldots,\{4,6\},\{5,6\}$, and in the next steps the five-element sets will be considered: $\{2,3,4,5,6\},\{1,3,4,5,6\}, \ldots,\{1,2,3,4,5\},\{0,3,4,5,6\}, \ldots,\{0,1$, $2,3,5\},\{0,1,2,3,4\}$. For each set $\left\{a_{0}, a_{1}, \ldots, a_{4}\right\}$ ten two-element subsets $\left\{a_{0}, a_{1}\right\},\left\{a_{0}, a_{2}\right\}$, $\ldots,\left\{a_{0}, a_{4}\right\},\left\{a_{1}, a_{2}\right\}, \ldots,\left\{a_{2}, a_{4}\right\},\left\{a_{3}, a_{4}\right\}$ are generated. So, as the final result, one obtains decomposition of the set $\{0,1, \ldots, 6\}$ into three subsets with cardinalities 3,2 , and 2 (note that 
the smallest numbers "occupy" the last subset at the start). There are $7 !(3 ! 2 ! 2 !)=5 \cdot 6 \cdot 7=210$ decompositions; a few of them are presented below:

$\begin{array}{lll}\{4,5,6\} & \{2,3\} & \{0,1\} \\ \{3,5,6\} & \{2,4\} & \{0,1\} \\ \{3,4,6\} & \{2,5\} & \{0,1\} \\ \{3,4,5\} & \{2,6\} & \{0,1\} \\ \ldots & \ldots & \ldots \\ \{0,2,5\} & \{3,4\} & \{1,6\} \\ \{0,2,4\} & \{3,5\} & \{1,6\} \\ \{0,2,3\} & \{4,5\} & \{1,6\} \\ \{4,5,6\} & \{0,1\} & \{2,3\} \\ \{1,5,6\} & \{0,4\} & \{2,3\} \\ \{1,4,6\} & \{0,5\} & \{2,3\} \\ \ldots & \ldots & \ldots \\ \{0,2,3\} & \{1,4\} & \{5,6\} \\ \{0,1,4\} & \{2,3\} & \{5,6\} \\ \{0,1,3\} & \{2,4\} & \{5,6\} \\ \{0,1,2\} & \{3,4\} & \{5,6\}\end{array}$

Note that decompositions are considered as different when subsets are identical but generated in a different order, e.g. $\{4,5,6\},\{2,3\},\{0,1\}$ and $\{4,5,6\},\{0,1\},\{2,3\}$.

Generated subsets are converted into spin configurations according with the rule: if $j$ belongs to the $i^{\text {th }}$ subset then a spin projection at $j^{\text {th }}$ node is $s-l, l=0,1, \ldots, m-1$ and $m=2 s+1$, The listed above decompositions give rise to the following spin configurations ( \pm stand for \pm 1 , respectively):

$$
\begin{aligned}
& |+,+, 0,0,-,-,-\rangle \quad 22110000 \\
& |+,+, 0,-, 0,-,-\rangle \quad 22110100 \\
& |+,+, 0,-,-, 0,-\rangle \quad 22100010 \\
& |+,+, 0,-,-,-, 0\rangle \quad 22100001 \\
& |-,+,-, 0,0,-,+\rangle \quad 02011102 \\
& |-,+,-, 0,-, 0,+\rangle \quad 0 \quad 2001012 \\
& |-,+,-,-, 0,0,+\rangle \quad 0 \quad 2000112 \\
& |0,0,+,+,-,-,-\rangle \quad 112220000 \\
& |0,-,+,+, 0,-,-\rangle \quad 10222100 \\
& |0,-,+,+,-, 0,-\rangle \quad 10222010 \\
& |-, 0,-,-, 0,+,+\rangle \quad 01100122 \\
& |-,-, 0,0,-,+,+\rangle \quad 000110022 \\
& |-,-, 0,-, 0,+,+\rangle \quad 000110122 \\
& |-,-,-, 0,0,+,+\rangle \quad 00001122
\end{aligned}
$$




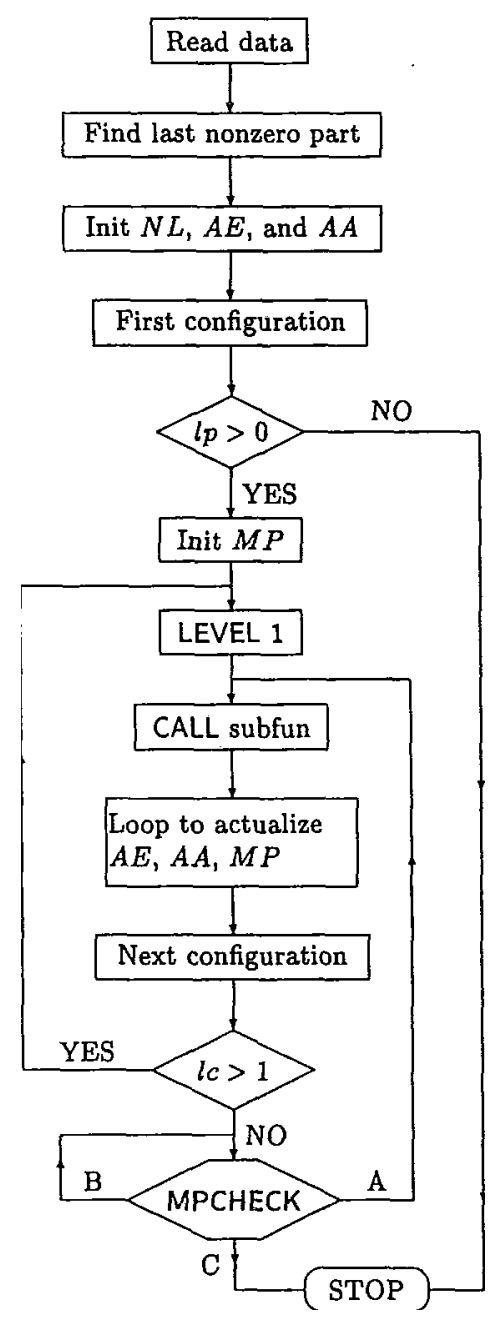

Fig. 5. Subsets (configurations) generation

In fact, to avoid negative and half-integer numbers, the program prints theses numbers increased by $s$, i.e. $2 s-1,1=0,1, \ldots, 2 s$, what is presented in the second column above for $\mathrm{s}=1$.

The above presented remarks suggest that, first of all, there should be arrays containing generation parameters ( $k, n, a, p p$ in the declaration of the function "subfun") depending on the level 1 and the level counter $1 c=m-1$ it self. These parameters are stored in arrays par[m], $N L[m], A A[m]$, and $M P[m]$, where $A A$ is in fact a two-index arrays since each row $A A[l c]$ contains a $\operatorname{par}[\mathrm{lc}]-e l e m e n t$ subset. However, the procedure "subfun" always works with a set $\{0,1, \ldots$, $N L[l c]-1\}$, whereas the actual elements may be different. In the above presented example this procedure generates at first two-element subsets of the set $\{2, \ldots, 6\}$, next of the set $\{1,3, \ldots, 6\}$ is considered, and so on till the last case - $\{0,1, \ldots, 4\}$. Therefore, it is necessary to introduce a two-dimensional array containing actual elements $(E)$ of the set in a question. The array par is a part of input data and the array $N L$ is determined at the start of a procedure. The other arrays 
$(A A, M P, E)$ are initialized and then modified during the generation process with use of a working array $\sec [N]$ containing sectioning points (it will described in more details below). The conversion from subsets to spin configurations is done by the formula

It should be read as follows:

$$
\operatorname{CON} F[E[I][A A[I][j]]]=I \text {. }
$$

1. For each level $i$ (related to spin projection $i-s$ ) relative indices of nodes are stored in a row $A A[i], 0 \leq j<k_{i}$;

2. The $i^{\text {th }}$ row of the array $E$ contains absolute, i.e. in the range $0, \ldots, N-1$, indices of these nodes;

3. At these nodes spin projections have the value $i-s$, so the appropriate elements of the array $C O N F$ equal $i$.

$01 \operatorname{lp}:=n$

02 repeat $l p:=l p-1$ until $\operatorname{par}[l p] \diamond 0$

$03 N L[0]:=\operatorname{par}[0]$

$04 l:=N-\operatorname{par}[0]$

05 for $I:=0$ to $N L[0]-1$ do $A E[0, I]:=l+I$

06 for $j:=1$ to $l p$ do begin

$07 \quad N L[j]:=N L[j-1]+\operatorname{par}[j]$

$08 \quad l=N-N L[j]$

09 for $I:=0$ to $N L[j]-1$ do $A E[j, i]:=l+i$

10 end

11 for $j:=0$ to $l p$ do

12 for $I:=0$ to $\operatorname{par}[i]-1$ do $A A[j, i]:=\ddot{i}$

13 print (conf)

Fig. 6. Initialization and generation of the first configuration

The algorithm starts with calculation of elements $N L[j]$ as

$$
N L[j]=\sum_{i=0}^{j} \operatorname{par}[i], \quad j=0,1, \ldots, l p,
$$

and the initialization of $A A$ and $E$ in such a way, that the first decomposition into subsets is $(i=0,1, \ldots, l p<m)$

$$
\begin{aligned}
& \{N-N L[0], N-N L[0]+1, \ldots, N-1\}, \\
& \ldots \\
& \{N-N L[i], N-N L[i]+1, \ldots, N-N L[i-1]-1\}, \\
& \ldots \\
& \{\operatorname{par}[l p], \operatorname{par}[l p]+1, \ldots, \operatorname{par}[l p-1]+\operatorname{par}[l p]-1\}, \\
& \{0,1, \ldots, \operatorname{par}[l p]-1\},
\end{aligned}
$$


what corresponds to the following configuration

printed as

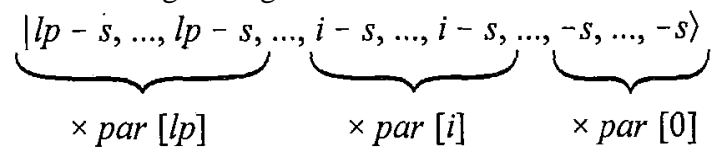

$$
\underbrace{l p \ldots l p}_{\times \operatorname{par}[l p] \times \operatorname{par}[i] \times p a r[0]} \cdots \underbrace{i \ldots i} \ldots \underbrace{0 \ldots 0} .
$$

It is realized by the following starting values of $\boldsymbol{E}$ and $\boldsymbol{A A}$ (the $i^{\text {th }}$ rows are presented):

$$
\begin{aligned}
& A E[i]=\left[N-N L[i], N-N L[i]+1, \ldots, N-1, *, \ldots,{ }^{*}\right], \\
& A A[i]=\left[0,1, \ldots, k_{i}-1,{ }^{*}, \ldots,{ }^{*}\right]
\end{aligned}
$$

(elements denoted by an asterisk "*" are irrelevant and they need not to be initialized).

The array MP containing "moving points" is initialized if and only if there are more than one part. The procedure works mainly at the level $l c=1$, i.e. it generates $\operatorname{par}[1]$-element subsets of

$N L[1]$-element set. Of course, at the same moment it generates $\operatorname{par}[0]$-element subset of $N L[0]-$ element set, since $\operatorname{par}[0]=N L[0]$. After generation of the next subset by the function "subfun" the array $A E$ has to be modified. It is done for all levels from $k=l c$ to 1 with the use of auxiliary array $\sec [N]$ constructed for each $k$ as

$$
\sec [i]=A A[k, i], \quad i=0,1, \ldots, \operatorname{par}[k]-1
$$

and

$$
\sec [\operatorname{par}[k]]=N L[k]
$$

(note that the element $A A[k, \operatorname{par}[k]]$ is not determined, but, if one prefers, the array sec may not be introduced). Elements of $A E$ are substituted consecutively, what is controlled by a variable $l$

(lines 24-30). For a given $k$ elements of $A E$ at the level $k-1$ are replaced by elements not used at the $k^{\text {th }}$ level. To illustrate this part of the proposed procedure let us consider the previously discussed partition $[3,2,2]$ at the moment when the level counter $l c=2$ (for $l c=1$ modifications of arrays are less visible). This value is reached when every tenth configurations is generated ( $\operatorname{since}(5 ! / 2 ! 3 !)=10)$. For example the $110^{\text {th }}$ configurations is $\left(\begin{array}{lllllll}0 & 2 & 0 & 0 & 1 & 1 & 2\end{array}\right)$ (see also the above presented example). The MPCHECK module, discussed below, leads switching to $l c=2$ so the actual parameters of the procedure "subfun" are as follows:

$$
\begin{aligned}
& \operatorname{par}[2]=2, \\
& N L[2]=7, \\
& A A[2]=[1,6], \\
& M P[2]=2 .
\end{aligned}
$$

Since the second element of $A A[2]$ has reached the $\operatorname{limit} N-1=6$ then the next generated subset is

$$
A A[2]=[2,3]
$$

and the moving point is changed to $M P[2]=1$. The arrays $A E, A A$, and $M P$ have to be modified for $k=2$, 1. At the first case the sectioning points are $0,2,3,7$ (the last three are stored in the 
array sec) and $l$ is set to 0 . The following subsets are considered (see lines 25, 28, and 29): $\{0,1\}$, $\varnothing$, and $\{4,5,6\}$. Since the pair $\{2,3\}$ is assigned to the last subset then the available elements for the next subset are $0,1,4,5,6$ and the row $A A[1]$ is changed from $[3,4]$ to $[0,1]$ (line 32). At the same moment the moving point $M P[1]$ is again set to $\operatorname{par}[1]=2$ (line 33). Now the same

14 if $(l p>0)$ begin $\}$

15 for $(i:=n-1)$ downto $0 M P[i]:=\operatorname{par}[i]$

16 LEV1:

$17 \quad l c:=1$

$18 M P[1]:=\operatorname{par}[1]$

19 FUNCALL:

$20 M P[l c]:=\operatorname{subfun}(\operatorname{par}[l c], N L[l c], A A[l c], M P[l c])$

21 for $k:=l c$ downto 1 do begin

22 for $i:=0$ to $\operatorname{par}[k]-1 \mathrm{do} \sec [i]:=A A[k, i]$

$23 \sec [\operatorname{par}[k]]:=N L[k]$

$24 \quad l:=0$

25 for $j:=0$ to $\sec [0]-1$ do begin

$26 \cdot A E[k-1, l]:=A E[k, j] ; l:=l+1$

27 end

28 for $i:=0$ to $\operatorname{par}[k]-1$ do

29 for $j:=\sec [i]+1$ to $\sec [i+1]-1$ do begin

$30 \quad A E[k-1, l]:=A E[k, j] ; l:=l+1$

31 end

32 for $i:=\operatorname{par}[k-1]-1$ downto 0 do $A A[k-1, i]:=i$

$33 M P[k-1]:=\operatorname{par}[k-1]$

34 end $\{$ loop over $k\}$

35 print $(\operatorname{con} f)$

36 if $(l c>1)$ goto LEV1

37 MPCHECK:

38 if $(M P[l c]>1)$ goto FUNCALL

39 if $(\mathrm{AA}[l c, \operatorname{par}[l c]-1]<(N L[l c]-1))$ goto FUNCALL

$40 \quad l c:=l c+1$

41 if $(l c \leq l p)$ goto MPCHECK

42 end $\{l p>0\}$

Fig. 7. Generation of the consecutive configurations 
procedure is carried out for $k=1$. Since the first two elements $(A A[1]=[0,1])$ are reserved for the subset labeled by 1 then the last three $(4,5,6)$ will be available for the subset labeled by 0 . After this modifications one obtains:

$$
\begin{gathered}
A E[0]=[4,5,6], \quad A E[1] \quad=[0,1,4,5,6], \quad A E[2]=[0,1,2,3,4,5,6] ; \\
A A[0]=[0,1,2], \quad A A[1]=[0,1], \quad A A[2]=[2,3] .
\end{gathered}
$$

According with (1) the generated configuration is

$$
|0,0,+,+,-,-,-\rangle \text { or } 1112220000 .
$$

The next call to "subfun" changes $A A[1]$ to $[0,2]$, so the sectioning points are 0,2 , and 5 . Therefore, the available elements in $A E[0]$ are those indexed by 1, 3, and 4 in $A E[1]$, so $A E[0]$ $=[1,5,6]$ and the generated configuration is

$$
|0,-,+,+, 0,-,-\rangle \quad \text { orl } 00222100 .
$$

The last part of the proposed procedure is devoted to control the current level. The main rule is that after generation at the level $l c>1$ the next configuration is generated at the first level (line 36). If $l c-1$ then some conditions are checked to decide whether all configurations at $l c=1$ have been generated. The first condition is $M P[\mathrm{lc}]>1$ since $M P[\mathrm{lc}]=1$ (line 38) means that the end might be reached. It is checked by the next condition which is not satisfied if the last element in the row $A A[l c]$ is equal (line 39) $N L[l c]$ - 1 ( $c f$. the standard version of the procedure "subfun" in $[3,4]$. In these cases the function "subfun" is called (the case A in the flow chart in Fig. 5). If all subsets at the level $l c$ have been generated then $l c$ is increased and the same conditions are checked (line 40 and 41 and the case B in Fig. 5), unless $l c>l p$, i.e. all levels have reached their limits (case $\mathrm{C}$ in the flow chart in Fig. 5).

A number of generated decompositions (configurations) is given by a polynomial coefficient $N ! / \prod_{j=0}^{m-1} k_{j}$ !, so it is very large even for small $N$. Moreover, the main purpose of the presented programs is applications to mesoscopic systems with $N \leq 30$. Therefore, the test runs and timings have been performed in such cases. In the test version of the algorithm the conversion (1) and printing blocks are removed. The results obtained on the Pentium $300 \mathrm{MHz}$ machine are presented in Table II. The timings are calculated as mean values of several millions runs for $N$ $=6$ and thousands for $N=18$. In addition, the partition $[8,8,8]$ of $N=24$ is also tested: $9,465,511,770$ configurations are generated in about four hours what results in speed about 650 thousands configurations per second. In general, time necessaiy to generate one configuration increase nearly linearly with increasing $N$. It is confirmed by test runs for $N=100$, where about 250 thousands configurations are generated in one second.

\section{FINAL REMARKS}

The combinatorial algorithms, presented in this paper, seem to work quite efficiently even for quite large values of $N$ and $m$. However, both of them are limited by the "combinatorial explosion". It especially concerns the generation of decompositions into subsets (interpreted as magnetic configurations), since polynomial coefficients grow very rapidly. For example, there are more than $5 \cdot 10^{12}$ configurations corresponding to the partition $\left[\begin{array}{lll}10 & 10 & 10\end{array}\right]$ and their generation will take more than four months (on the Pentium $300 \mathrm{MHz}$ PC). Moreover, to analyze 
the ground state of an antiferromagnetic system consisting of $N=30$ spins $s=1$ one should consider all (non-ordered) partitions $\left[k_{0}, k_{1}, k_{2}\right]$ with $k_{0}=k_{1}[1]$. Furthermore, this step is only the beginning of a long process: configurations have to be stored and decomposed into orbits, matrix elements of operator matrices have to be determined and eigenproblems solved. On the other hand, systems with $s=1$ and $N \leq 20$ lead to several millions configurations for each partition $\left(133,024,320\right.$ for $\left.\left[\begin{array}{ll}7 & 7\end{array}\right]\right)$, so the generation process takes less than five minutes (assuming $0.5 \cdot 10^{6}$ configurations per second). Each configuration $\left.\mid m_{0}, m_{1}, \ldots, m_{N-1}\right)$ can be stored as a number a $=\Sigma_{j=0}^{N-1}\left(m_{j}+s\right) \cdot(2 s+1)^{j}$, i.e. a configuration is considered as a number written in the base $(2 s+1)$. In the presented example, $N=20$ and $s=1$, the maximal value of $a$ equals $3^{20}-1=3,486,784,400<2^{32}-1$, so one needs four bytes per configurations, what results in more than $500 \mathrm{MB}$ for the partition [7 7 6]. It is a very large number, but all configurations can be stored on a hard disk used in PC's. It seems, however, that the reasonable limit is $N=18$, where number of configurations is about seven times smaller (e.g. 17,153,136 for [6 6 6 ]). Larger spin values decrease this limit: a number of configurations reaches $15 \cdot 10^{6}$ for $N=15$ when $s=3 / 2$ and so on, but $4^{15}<2 \quad$ so again four bytes is enough to store one configuration. Such limits allow to investigate most of mesoscopic magnetic rings [6], what is the main purpose of the presented algorithms.

Table II. Number of configurations $N C$ and generation speed (configurations per second) for $N=6$ and $N=18$

\begin{tabular}{|c|c|c|c|c|c|}
\hline \multicolumn{6}{|c|}{$N=6$} \\
\hline$[k]$ & {$\left[\begin{array}{ll}5 & 1\end{array}\right]$} & [4 2] & $\left.\begin{array}{lll}4 & 1 & 1\end{array}\right]$ & {$\left[\begin{array}{ll}3 & 3\end{array}\right]$} & {$\left[\begin{array}{lll}3 & 2 & 1\end{array}\right]$} \\
\hline$N C$ & 6 & 15 & 30 & 20 & 60 \\
\hline speed & $1,597,444$ & $1,348,072$ & $1,390,691$ & $1,315,011$ & $1,323,714$ \\
\hline$[k]$ & {$\left[\begin{array}{llll}3 & 1 & 1 & 1\end{array}\right]$} & {$\left[\begin{array}{lll}2 & 2 & 2\end{array}\right]$} & {$\left[\begin{array}{llll}2 & 2 & 1 & 1\end{array}\right]$} & {$\left[\begin{array}{llllll}2 & 1 & 1 & 1 & 1\end{array}\right]$} & {$\left[\begin{array}{llllll}1 & 1 & 1 & 1 & 1 & 1\end{array}\right]$} \\
\hline$N C$ & 120 & 90 & 180 & 360 & 720 \\
\hline speed & $1,428,163$ & $1,435,933$ & $1,401,214$ & $1,407,239$ & $1,364,670$ \\
\hline \multicolumn{6}{|c|}{$N=18$} \\
\hline$[k]$ & {$\left[\begin{array}{lll}9 & 9 & 0\end{array}\right]$} & {$\left[\begin{array}{lll}9 & 8 & 1\end{array}\right]$} & {$\left[\begin{array}{lll}9 & 7 & 2\end{array}\right]$} & {$\left[\begin{array}{lll}9 & 6 & 3\end{array}\right]$} & {$\left[\begin{array}{lll}9 & 5 & 4\end{array}\right]$} \\
\hline$N C$ & 48,620 & 437,580 & $1,750,320$ & $4,084,080$ & $6,126,120$ \\
\hline speed & 596,272 & 623,760 & 653,922 & 688,761 & 789,093 \\
\hline$[k]$ & {$\left[\begin{array}{llll}1 & 2 & 6 & 0\end{array}\right]$} & {$\left[\begin{array}{llll}1 & 2 & 5 & 1\end{array}\right]$} & {$\left[\begin{array}{llll}1 & 2 & 4 & 2\end{array}\right]$} & {$\left[\begin{array}{llll}1 & 2 & 3 & 3\end{array}\right]$} & {$\left[\begin{array}{llll}1 & 6 & 1 & 1\end{array}\right]$} \\
\hline$N C$ & 18,564 & 111,384 & 278,460 & 371,280 & 306 \\
\hline speed & 632,461 & 716,757 & 770,845 & 832,466 & 849,292 \\
\hline
\end{tabular}

The problem of generation all decompositions of $N$-element set into subsets with cardinalities given by an ordered partition $\left[k_{0} k_{1}, \ldots k_{m-1}\right]$ has been solved in such a way that there is no recurrence, since sometimes it is difficult to realize recurrent algorithms. It should be underlined that in the proposed procedure all $k_{1}$-element subsets of $N L[1]$-element subset are generated many times (strictly speaking $-N ! /\left(k_{0}+k_{1}\right) ! \prod_{j=2}^{m-1} k_{j}$ ! times). To avoid this feature one can store all 
decompositions of $N L[1]$-element set into $k_{0}$ - and $k_{1}$-element subsets and then use stored results for the further generations at levels $l c>1$. However, it give rise to a problem of efficient storing and recalling of configurations. Moreover, the actual form of the algorithm is more flexible: the array $A E$ may contain any elements (for example, an element $A E[i][j]$ can be interpreted as an index of an array with actual objects in question) or the generation can be limited to subsets with given properties. It is worth noting that consecutively generated decompositions may differ considerably, e.g. $\left\{\begin{array}{lll}0 & 2 & 3\end{array}\right\}\left\{\begin{array}{llll}4 & 5\end{array}\right\}\left\{\begin{array}{lll}1 & 6\end{array}\right\}$ and $\left\{\begin{array}{lllllll}4 & 5 & 6\end{array}\right\}\left\{\begin{array}{llll}0 & 1\end{array}\right\}\left\{\begin{array}{lll}2 & 3\end{array}\right\}$ in the example presented in the main text. It seems interesting to construct an algorithm which generates decompositions with the minimal difference, when consecutive decompositions differ only by one interchange of two elements between two subsets, i.e. a decomposition in a form

$$
\{\ldots\} \quad \ldots\{\ldots a \ldots\} \quad \ldots\{\ldots b \ldots\} \quad \ldots\{\ldots\}
$$

is always followed by a decomposition obtained by swapping $a$ and $b$ only:

$$
\{\ldots\} \quad \ldots\{\ldots b \ldots\} \quad \ldots\{\ldots a \ldots\} \quad \ldots\{\ldots\} .
$$

Such algorithm is known for $m=2$ [3,4], i.e. for generation of $k$-element subsets.

\section{Acknowledgments}

This work is supported by the Polish State Committee for Scientific Research (KBN) within the project No. 8 T11F 027 16. The author is indebted to Prof. G. Kamieniarz for inspiring and valuable discussions. Some of numerical calculations were carried out on SGI Power Challenge in the Supercomputing and Networking Center in Poznań.

\section{References}

[1] S. Bucikiewicz, W. Florek, CMST, this volume.

[2] G. Kamieniarz, R. Matysiak, W. Florek, S. Walcerz, J. Magn. Magn. Mater., 203, 271 (1999).

[3] E. N. Reingold, J. Nivergelt, N. Deo, Combinatorial Algorithms. Theory and Practice, Prentice Hall, Engelwood Cliffs NJ, 1977.

[4] W. Lipski, Combinatorics for Programmers. Polish Sci. Publ. (PWN), Warsaw, 1982 [in Polish].

[5] A. Kerber, Algebraic Combinatorics via Finite Group Actions. B I Wissenshaftsverlag, MannheimWien-Zürich, 1991.

[6] D. Gatteschi, A. Caneschi, L. Pardi, R. Sessoli, Science, 265, 1054 (1994); A. Lascialfari, D. Gatteschi, A. Cornia, U. Balucani, M. G. Pini, A. Rettori, Phys. Rev. B, 57, 1115 (1998);

A. Caneschi, D. Gatteschi, C. Sangregorio, R. Sessoli, L. Sorace, A. Cornia, M. A. Novak, C. Paulsen, W. Wernsdorfer, J. Magn. Magn. Mater., 200, 182 (1999). 